\title{
Optimization OF THE RECURSIVE ONE-SIDED Hypothesis Testing Technique for Autonomous Threshold Estimation in Cognitive Radio
}

\author{
James Odinaka Okonkwo ${ }^{1}$ and Sylvester Ajah ${ }^{2}$ \\ ${ }^{1}$ Department of Telecommunication Engineering, Federal University of Technology \\ Minna, Niger State, Nigeria \\ ${ }^{2}$ Computer Engineering Technology, AkanuIbiam Federal Polytechnic Unwana, Ebonyi \\ State, Nigeria
}

\begin{abstract}
In this paper, an optimized Recursive One-Sided Hypothesis Testing (ROHT) threshold estimation algorithm for energy detection based on Cognitive Radio (CR) application is presented. The ROHT algorithm is well known to compute and correct threshold values based on the choice of the parameter values; namely the coefficient of standard deviation (z-value) and the stopping criteria ( $\beta$ ). A fixed computational process has been employed in most cases to estimate these parameter values, thus rendering them non-adaptive under different sensing conditions. Also, this fixed (manual tuning) process requires prior knowledge of some noise level to enable pre-configuration of a predefined target false alarm rate. This renders the parameter estimation process cumbrous and unworkable for real-time purposes, particularly for autonomous CR applications. Furthermore, using wrong parameter values may lead to either too high or too low false alarms or detection rates of the algorithm. Sequel to aforementioned mentioned constraints, we propose a new mechanism for instantaneous parameter optimization of the ROHT algorithm using Particle Swarm Optimization (PSO) algorithm. Our PSO-ROHT model design was extensively tested under different conditions and results were compared to the non-optimized ROHT. The results obtained show that the proposed design effectively adapts the parameter values of the Recursive One-Sided Hypothesis Testing algorithm in accordance with the input dataset under consideration. Also, that the proposed optimized model outperforms its non-optimized counterpart following the estimated detection probability and false alarm probability of both schemes, particularly in detecting Orthogonal Frequency-Division Multiplexing signals. In detecting the Orthogonal Frequency-Division Multiplexing signals at signal-to-noise ratio of $3 \mathrm{~dB}$ and above, the proposed model achieved a higher detection rate of 96.23\% while maintaining a low false alarm rate below 10\%, which complies with the IEEE 802.22 standard for Cognitive Radio application. Our PSO-ROHT algorithm is shown to be highly effective for autonomous and full blind signal detection in $C R$, with strong potentials for application in other areas requiring automatic threshold estimation.
\end{abstract}

\section{KEYWORDS}

Adaptive, Autonomous, Cognitive Radio, Energy detector, Optimization, Particle Swarm, PSO - ROHT algorithm, ROHT, threshold

DOI: 10.5121/ijwmn.2018.10601 


\section{I .INTRODUCTION}

A cognitive radio (CR) is a form of wireless communication technology in which a transceiver can intelligently detect which communication channel is free or not, and instantly engage the vacant channels while avoiding occupied ones to prevent interference [1][2]. This mechanism optimizes the use of the available Radio-Frequency (RF) spectrum for the unlicensed or secondary user (SU), while minimizing the interference level experienced by the licensed user (LU) or primary user (PU).The IEEE 802.22 draft standard for Wireless Regional Area Network (WRAN) using white spaces, lays particular emphasis on using Spectrum Sensing(SS) for spectrum identification in CR [3].The aforementioned standard is aimed at using CR techniques to allow sharing of geographically unused spectrum allocated to the television broadcast service, on a non-interfering basis, to bring broadband access to hard-to-reach, low population density areas and rural environments. The CR identifies spectrum in the environment using SS.

Several methods have been proposed for SS namely, the Interference Temperature Detection method, the Matched Filter Detection method, the Cyclostationary Feature Detection method, and the Energy Detector (ED) method. However, to determine which choice of SS technique to best drive a CR, the following factors have to be put into consideration. These factors include; the desired accuracy, sensing duration, network requirement and the computational complexity. A summary of the SS techniques is shown in table 1 as presented in [4].

Table 1: A summary of SS Techniques [4]

\begin{tabular}{|c|c|c|}
\hline $\begin{array}{l}\text { Spectrum sensing } \\
\text { technique }\end{array}$ & Advantages & Disadvantages \\
\hline $\begin{array}{l}\text { Energy Detector } \\
\text { (ED) }\end{array}$ & $\begin{array}{l}\text { The computational power requirements is very } \\
\text { low } \\
\text { Low complexity. } \\
\text { It is easy to deploy } \\
\text { Independent of the primary user (PU) waveform. }\end{array}$ & $\begin{array}{l}\text { It is highly dependent on the variation of noise. } \\
\text { Performs Poorly in environments with low SNR. } \\
\text { It has a longer detection time }\end{array}$ \\
\hline $\begin{array}{l}\text { Matched Filter } \\
\text { detection }\end{array}$ & $\begin{array}{l}\text { Best in white Gaussian noise. } \\
\text { Shorter sensing duration }\end{array}$ & $\begin{array}{l}\text { Needs earlier information of the primary user } \\
\text { signal } \\
\text { It requires additional equipment for coordination } \\
\text { with the Primary User }\end{array}$ \\
\hline $\begin{array}{l}\text { Cyclostationary } \\
\text { Feature Detection }\end{array}$ & Resilient to variations in the noise levels & $\begin{array}{l}\text { It is very intricate and requires high } \\
\text { computational ability at the nodes }\end{array}$ \\
\hline $\begin{array}{c}\text { Interference } \\
\text { Temperature } \\
\text { Detection } \\
\end{array}$ & $\begin{array}{l}\text { It sets predetermined interference limit which it } \\
\text { uses to shield the PU from interference }\end{array}$ & $\begin{array}{c}\text { It is required to know the PU's location. } \\
\text { High computational power is required at its } \\
\text { nodes }\end{array}$ \\
\hline
\end{tabular}

The Energy Detector (ED) is considered the most viable technique mainly for its ease of deployment, low computational power, low complexity and its independence of the Primary User (PU) signal waveform. Typically, the ED employs a threshold value to decide if PU signals are present or not in a given channel [5]. In most designs, the threshold value of an ED is determined by the known noise level in the system [5]. However, noise levels are random in nature with ambient values that are often cannot be theoretically be deduced by the detector, thus making threshold estimation a challenge.

As a result, newer ED designs are required to adapt their respective threshold values in accordance with varying channel conditions. This has led to the design of several adaptive threshold estimation techniques (ATT) in the literatures[6][7][8][9], with the Recursive One-Sided Hypothesis Testing (ROHT) algorithm standing out as a type of ATT that has been popularly 
proposed for the ED[4][8][9][10]. The ROHT is known for its simplicity, effectiveness and efficiency [10]. However, the effectiveness of the ROHT algorithm depends on the choice of its parameter values. In previous work[4], the performance of the ROHT algorithm was enhanced with noise variance estimation and a constant false alarm rate (CFAR) was employed to act as a defence mechanism against interference of the PU at low signal to noise ratio (SNR). However global parameter values have not yet been obtained because of the unstable nature of most spectral conditions and poor choice of the ROHT algorithm's parameter values may lead to either too high or too low threshold values, resulting in either too high or too low false alarm and detection rates of the algorithm[4].

To address the above existing limitations, we propose a new and automatic approach for optimizing the ROHT algorithm's parameter values based on the Particle Swarm Optimization (PSO) algorithm. The PSO algorithm determines the optimal parameter values of the ROHT algorithm per input dataset. This new mechanism optimizes the ROHT algorithm's parameter values without prior knowledge of the spectral condition under consideration. Our PSO-ROHT model was developed and extensively tested to ascertain its performance limits. Results obtained shows that the PSO-ROHT model provides full blind spectrum sensing with appreciable performance levels to improve SS in CR.

The rest of this paper is structured as follows; section II discusses related works, III energy detector system, IV ROHT algorithm, V particle swarm optimization algorithm, VI the PSO ROHT model, VII results and discussions and section VIII conclude the work with further works.

\section{RELATED WORKS}

The concept of Cognitive Radio (CR) was first introduced by Joseph Mitola III in 1999 in his paper, [11].A cognitive radio can be described as an intelligent radio that can be programmed and configured dynamically to adjust its transmitter parameters based on the interaction with the environment it operates in [4]. The fundamental aim of cognitive radio is to allow users who do not have spectrum rights to temporarily operate in the unused portions of the licensed spectrum as Secondary Users (SU). Cognitive radio employs the spectrum overlay technique to dynamically access the unused portions of the licensed spectrum that are otherwise known as spectrum holes or white spaces, as shown in figure 1[4]. The main aim of cognitive radio is to obtain the best available spectrum band through cognitive ability and re-configurability. Cognitive ability is the capacity of the secondary users to sense the radio conditions in its immediate environment such as the presence of a licensed user, transmission frequency and power, bandwidth and modulation scheme. However, this capability cannot solely depend on monitoring the power levels in selected frequency bands. Advanced techniques such as autonomous learning and action decision have to be employed to assess and capture the temporal and spatial variations in the radio environment so as to minimize interference to the licensed users. On the other hand, the SUs are able to make decisions and adapt their operation parameters based on the conditions in the radio environment due to their re-configurability. Furthermore, CRs can be programmed to operate in a number of frequency bands using a variety of access technologies as permitted by the hardware design [12]. The CR identifies spectrum in the environment using SS. 


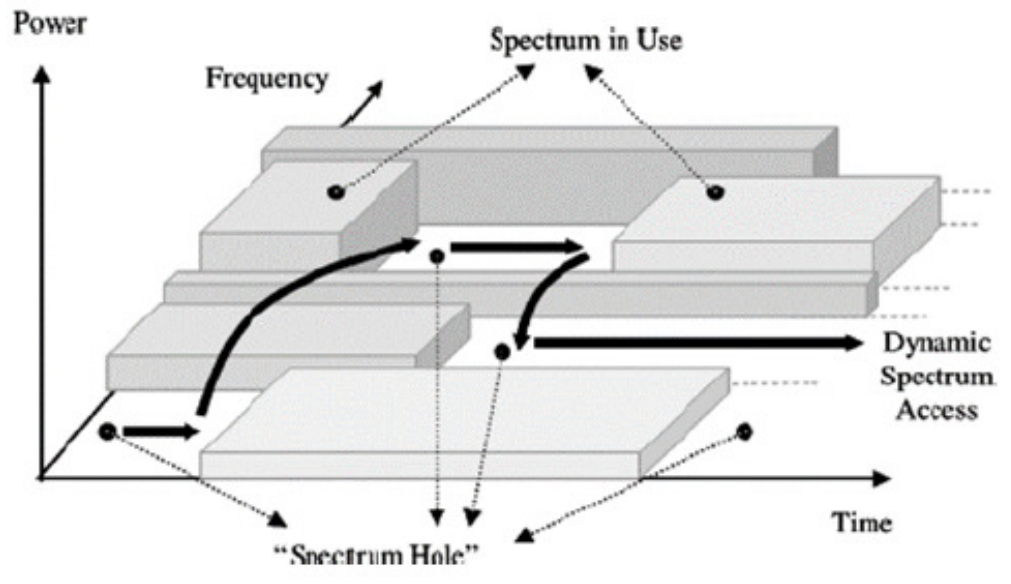

Figure 1: Spectrum Holes

Energy detection is considered the most viable technique for SS in CR, and ROHT algorithm has stood out as a type of ATT that has been popularly used in the ED for threshold estimation. The ROHT was first proposed by F. Weidling et al. [9], it is a statistical threshold selection technique based on the mathematical technique for analyzing random noise referred to as one-sided hypothesis testing. The ROHT algorithm as applied in the context of ED tries to identify the signal components from the noise components of the received signal and subsequently set the decision threshold. Efforts have been made to enhance the ROHT algorithm with an example found in [4]. These efforts focused on improving the computation for the threshold and the localization capacity of the ROHT algorithm. Despite these, the ROHT algorithm remains limited by certain challenges like, lack of global parameter values for the algorithm need for prior knowledge of some noise level to set a target false alarm rate and its poor performance in low SNR.

Following the review, it is essential to understand the implications of each parameter as it pertains to the performance of the ROHT algorithm. These are explained as follows;

a) Z-value: This denoted the coefficient of standard deviation. It is the number of standard deviations away from the mean in a distribution. It is related to the confidence level. It directly affects the decision threshold. Too high value of the $\mathrm{z}$-value parameter leads to too high threshold value resulting in high miss detection rate, while too low value of the z-value parameter leads to too low threshold value resulting in high false alarm rates.

b) $\beta$ : This represents the stopping criteria. It is an arbitrary positive value that is specified. The ROHT algorithm stops iterating when the change in the standard deviation between two consecutive iterations becomes less than or equal to $\beta$. Its stated as $(\sigma i+1-\sigma i) \leq \beta$.

Essentially, the choice of these parameter values determines how well the ROHT algorithm will perform. Since its inception [9], the ROHT's parameter values have been computed via the use of different theoretical models which are independent of the data under consideration. Due to this independence, it remains difficult to establish a particular global value for each parameter of the ROHT algorithm to guarantee optimum performance in all spectral environments. Consequently, the ROHT algorithm cannot be fully automated thus requiring human input to manually reconfigure these parameter values whenever sensing conditions considerably deviate from preset conditions. In addition, poor choice of these parameter values, which may occur when sensing 
conditions abruptly change may lead to poor performance of the ROHT algorithm. This may lead to either too high false alarm rates, or too high miss detection rates.

In [13], the authors proposed an adaptive threshold method for SS in multi - channel cognitive radio networks. The proposed method which does not need estimation of noise variance was targeted at reducing the effects of impairments inserted by wireless channel and non - stationary noise. Their results show that adaptive threshold has low false alarm and missed detection requirements of multi - channel cognitive radios when the $\mathrm{z}-$ value is selected properly for either narrow or wideband SS. The research output though commended, but it still requires manual selection of z-value.

Thus, it is essential to develop new methods to establish appropriate values for these parameters in an automatic manner. This will greatly enhance the potentials of the ROHT algorithm resulting in a fully automatic approach for possible application in diverse areas including pattern recognition, image processing, industrial processes, and in future intelligent systems (robotics). Motivated by the above, we make a first attempt in this paper to provide a novel mechanism for computing the ROHT parameter values automatically on the fly (implying per dataset). We enhance the ROHT algorithm into a more sophisticated toolset capable of diverse applications in autonomous systems.

\section{ENERGY DETECTOR SYSTEM}

The energy detection system is represented in figure 2. Typically, we consider the reception of a Radio Frequency (RF) signal emanating in the radio environment. These signals are received at the front end of the detection system by an antenna designed to operate within a specified frequency range(s), for example, within the VHF/UHF band to detect TV white spaces. The received continuous waveforms, $y(t)$, are passed into the energy estimator block where filtering and analogue to digital conversion takes places. To obtain the frequency domain version of the input signals, the energy estimator block computes the Discrete Fourier Transformation (DFT) of the signal. It then conducts a squaring operation and an averaging function to obtain $\mathrm{Y}(n)$.

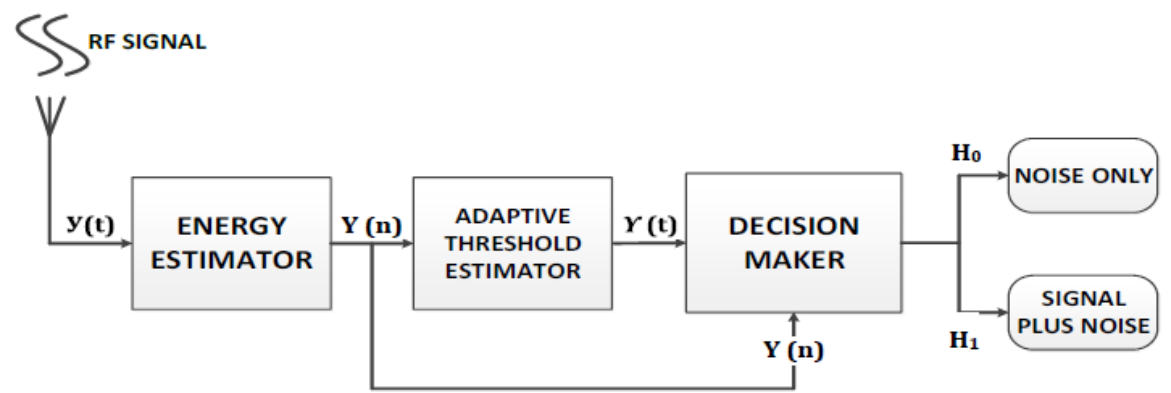

Figure 2: The Energy Detector System

The energy samples, $Y(n)$, are considered to be the test statistic in this case. These samples are passed into the adaptive threshold estimator block to dynamically compute an appropriate threshold value, $\gamma(T)$; which is a function of a certain sensing period, $T$. The test statistic, $Y(n)$, is compared to $\gamma$, to determine the state of the channel. If the channel is vacant $(Y(n),<\gamma)$, then $H_{0}$ is declared implying that the channel contains only noise samples, and if the channel is occupied $(Y$ $(n), \geq \gamma)$, then $H_{1}$ is declared implying the presence of signal plus noise in the channel. These hypotheses are generally defined as: 


$$
\begin{aligned}
& H_{0}: Y(n)=W(n), \quad \text { for } n=1,2, \ldots, N \\
& H_{1}: Y(n)=X(n)+W(n), \quad n=1,2, \ldots, N
\end{aligned}
$$

Where $n$ denotes the frequency sample index, $N$ is the total number of frequency samples, $X(n)$ represents the transmitted PU signal, $W(n)$ is modeled as Additive White Gaussian Noise (AWGN), and $Y(n)$ denotes the energy of the received signal at the output of the energy estimator. In this case, the total number of samples can be estimated using (3).

$$
N=2^{[(\log 2(T * f s)-1)]}
$$

Where $T$ is the total sensing period and $f_{s}$ is the sampling frequency.

Consequently, the entire detection process is expected to determine either $H_{0}$ or $H_{1}$; which strongly depends on the choice of $\gamma$. Thus, developing effective methods for estimating the value of $\gamma$ in an adaptive and autonomous manner, without prior knowledge of the noise level in the band will form the focus of subsequent subsections in this paper.

\section{ROHT AlgORITHM}

The Recursive One-Sided Hypothesis testing (ROHT) algorithm is considered for use in the adaptive threshold block of Figure 2. As noted in Section 1, the ROHT algorithm is considered for its simplicity, effectiveness and efficiency. In this section, we describe the algorithmic process of the ROHT algorithm, which we shall henceforth refer to as the non-optimized ROHT algorithm. The flow chart of the non-optimized ROHT threshold computation process is presented in Figure 3.

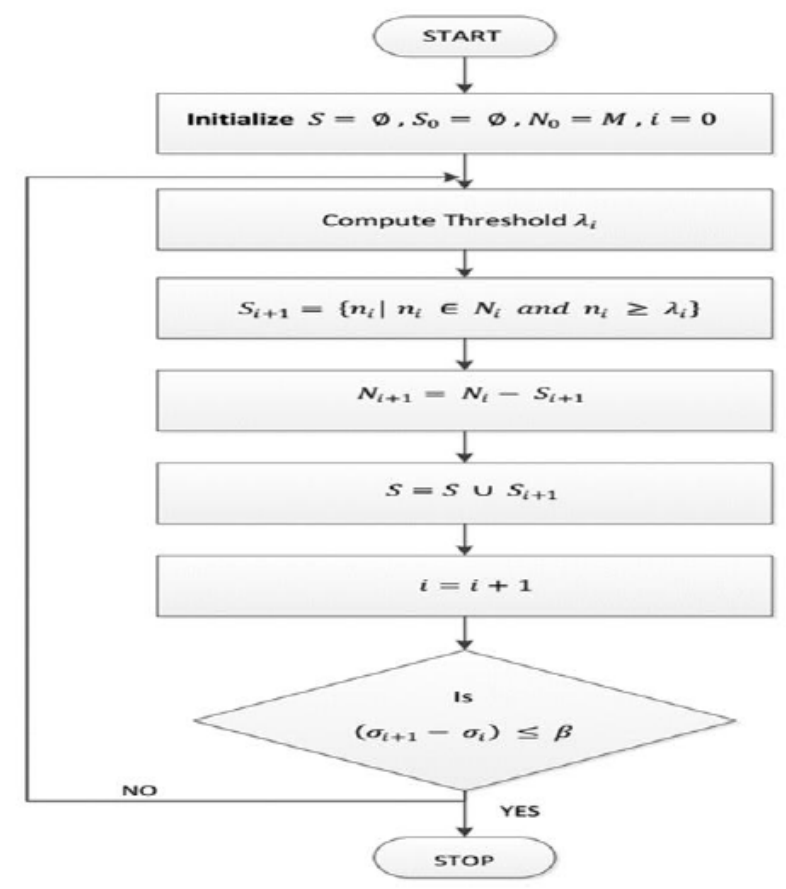

Figure 3: The Non-optimized ROHT Algorithm 
The algorithm first begins by initializing the set of signal components within the received measurements and should not be a null set. At the start of the algorithm, it is assumed that the received measurements contain more noise components than signal components and thus the purpose of the recursive one side hypothesis testing is to disprove this null hypothesis. The algorithm then proceeds to set the initial decision threshold which is given as a function of the zvalue and the standard deviation and the mean of the components in the $i$ th iteration. Based on the $(z$-value $)$ and the decision initial threshold, the algorithm then views a given percentage of measurements on the right-hand side of the normal Gaussian distribution as signal components and considers everything to the left-hand side as noise components. The identified signal portions are discarded and the process repeats. The algorithm comes to a halt once the difference in standard deviation between two consecutive iterations is less than a specified random positive value given as $(\beta)$. The generated threshold, mean and standard deviation are considered to be the optimal and accurate for the entire frequency band under consideration.

\section{V.PARTicle Swarm Optimization Algorithm}

Many optimization algorithms exist in literatures; however, we considered the Particle Swarm Optimization (PSO) algorithm particularly for its fast and efficient performance, use of few parameters, ease of parallelizing for concurrent processing and simplicity. It is an optimization technique that optimizes a problem by iteratively trying to improve a candidate solution, with regards to a given measure of quality. It was inspired by the social behavior of bird flocking and fish schooling. Thus, we sought to incorporate the PSO with the ROHT into the adaptive threshold estimator block of the ED in figure 2for spectrum sensing in CR, while ensuring speed and accuracy of the overall model.

The PSO algorithm using particle position update rule was applied in our model for finding the best global position, as a solution to our model using (4).

$$
P_{i}^{(t+1)}=P_{i}^{t}+V_{i}^{(t+1)} V_{i}(t+1)
$$

Where ${ }^{(t+1)}$ denotes the new particle updated position for a particle. Using particle update function, $P i t$ is the particle's present position and $V_{i}^{(t+1)}$ is the updated particle's velocities. This function in (4) helps to move the particle to their new position. The PSO concept can be described as swarm of particles in a search space, each particle updating its current position and comparing its personal position and velocity to the entire swarm's global best position, as a potential better solution to replace the non-global best solution in the search space.

The aim is to use the particles adaptation, to get the optimal parameter values for the ROHT algorithm, using the set objective function. The basic algorithm steps involved in the PSO are presented in Table 2. 
International Journal of Wireless \& Mobile Networks (IJWMN) Vol. 10, No. 6, December 2018

Table 2: The PSO Algorithm Steps

\begin{tabular}{|c|c|}
\hline 1 & $\begin{array}{l}\text { The agents (particles) population is spread evenly over } \mathrm{X} \text {. where } \mathrm{X} \text { is the number of particles, usually } \\
\text { from } 1 \text { to } 50 \text {. }\end{array}$ \\
\hline 2 & Evaluate the position of each particle in accordance with the set "objective function" \\
\hline 3 & Update the particle's current position if it is better than its previous best. \\
\hline 4 & Ascertain the best particle(according to the best position of the particle) \\
\hline 5 & $\begin{array}{l}\text { Update the velocities of the particle using; } \\
\qquad \mathrm{V}_{\mathrm{i}}{ }^{(\mathrm{t}+1)}=\mathrm{V}_{\mathrm{i}}^{\mathrm{t}}+\mathrm{C}_{1} \mathrm{U}_{1} \mathrm{t}\left(\mathrm{Pb}_{1}-\mathrm{P}_{1}^{\mathrm{t}}\right)+\mathrm{C} 2^{*} \mathrm{U}_{2} \mathrm{t}\left(\mathrm{gb}_{1}-\mathrm{P}_{1}^{\mathrm{t}}\right) \\
\text { Where } \mathrm{V}_{\mathrm{i}}^{\mathrm{t}} \text { is inertia, } \mathrm{C}_{1} \text { and } \mathrm{C}_{2} \text { are weight of local information and global information respectively, } \mathrm{Pb} \\
\text { is particle's personal best position, } \mathrm{P} \text { is the particle's position and } g b \text { is the global best position of the } \\
\text { swarm, } \mathrm{U}_{1} \text { and } \mathrm{U}_{2} \text { are random variables. }\end{array}$ \\
\hline 6 & Move particle to their new position using $\mathrm{P}_{1}{ }^{(\mathrm{t}+1)}=\mathrm{P}_{\mathrm{i}}{ }^{\mathrm{t}}+\mathrm{V}_{\mathrm{i}}{ }^{(\mathrm{t}+1)}$ \\
\hline 7 & Move to step 2 until terminating criteria is met. \\
\hline
\end{tabular}

\section{THE PSO-RoHT MODEL}

Our PSO-ROHT model is shown in figure 4. The model takes the energy samples estimated by the energy estimator block (see figure4) as input. These samples are fed into the ROHT algorithm. By using the PSO component of our model, initial parameter values (IPV) are set and fed into the ROHT algorithm. The ROHT algorithm uses these initial assigned parameter values to estimate an initial threshold value (ITV). The ITV is evaluated by the PSO algorithm using the designed objective function. The PSO algorithm iterates via its well-known processes toward estimating the final optimal ROHT parameter values (RPV). These optimized values are then fed into the ROHT algorithm, where defined iterative steps ensue toward an optimal threshold value (RTV), . The final estimated threshold value is then fed into the decision maker block to decide on the presence/absence of PU signals in the band.

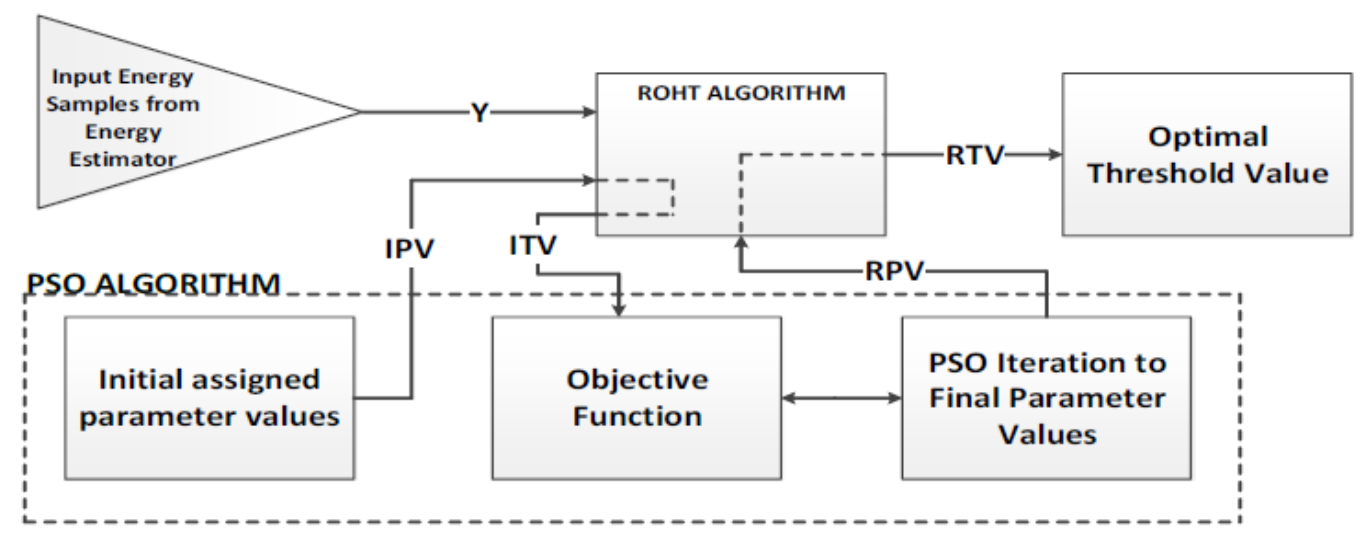

Figure 4: Our proposed PSO-ROHT Model for the Automatic, Dynamic and Optimized Estimation of Effective Threshold Values for Energy Detection in CR. 
A. PSO-ROHT Objective Function: The success of any optimization task depends on the design choice of the objective function. In this work we adopted the within-class variance function as in (5) by Otsu in [14]. The model was proposed in order to evaluate the "probity" of the threshold value of gray-level image, based on the discriminant analysis principle. We used the within-class variance model because of its simplicity and effectiveness. The use of this Otsu's model to enhance the ROHT algorithm has never been done before. In this work, the fitness function is given as;

$$
\sigma(\gamma)=\left[\left(P_{s} * \sigma^{2} s\right)+\left(P_{n} * \sigma^{2} n\right)\right]
$$

Where, $\sigma(\gamma)$ is the within-class variance computed as a function of the threshold value, $\gamma$, estimated by the ROHT algorithm, $P_{s}$ is the probability of the class of estimated signal samples as a function of the threshold $\gamma, P_{n}$ is the probability of the class of estimated noise samples, $\sigma_{2 s}$ is the variance of the class of estimated signal samples, and $\sigma_{2 n}$ is the variance of the class of estimated noise samples, all computed as a function of the threshold value estimated by the ROHT algorithm. Eqn. 5 is typically computed for different threshold values estimated via the ROHT algorithm, and the minimization function for obtaining the final optimal threshold value is given in (6).

$\sigma(\gamma *)=\min \sigma(\gamma)$

Where $\gamma^{*}$, is the optimal threshold value.

\section{B. PSO-ROHT Operational Process:}

a) Input Data: The process of the PSO-ROHT model (in figure 4) begins with receiving the input energy sample dataset. This is done by using the "load dataset" command.

b) PSO parameter Definition/Settings: The PSO algorithm is engaged by setting its optimization operating parameters such as the number of particles, $\mathrm{P}$, the number of iteration, (iter_num), number of parameters to optimize, dim, PSO's weight of local and global information, $\mathrm{C}$ for $\mathrm{C}_{1}$ and $\mathrm{C}_{2}$, PSO's momentum of inertia, $\mathrm{W}$ and the number of runs. Our PSO-ROHT model, operational values were chosen thus; $\mathrm{P}=50$, (iter_num) $=50$, dim $=2$, $\mathrm{C}=1, \mathrm{~W}=0.3$ and runs $=2$. These values were arrived at after several extensive simulations conducted using the PSO-ROHT model.

c) ROHT parameter Constraint Definition: The limits of the ROHT parameters to be optimized are set. In section 3, we noted the ROHT parameters to be the (z-value) which is the coefficient of standard deviation and the $(\beta)$, which is the stopping criteria of the algorithm. These parameters were assigned upper and lower limits. Also, the PSO-ROHT model considers only two parameters for optimization, resulting in a dimension of $\operatorname{dim}=2$. The constraints are thus appended as follows: For the z-value we have ( $z_{-} u p$ and $\left.z_{-} d o w n\right)$ and for the $\beta$ we have (Stop_up and Stop_down).

d) PSO parameter initialization: The parameters of the PSO such as the current position of the particle and velocity of the particles are initialized as regards to the set limits of the ROHT parameter constraint. 
e) Evaluation of the Fitness Function: This stage evaluates the fitness (objective) function of the PSO-ROHT, in order to find the optimal ROHT parameter values. The ROHT algorithm estimates both the initial and subsequently final threshold value, $\gamma$ while been controlled by the PSO algorithm. This control refers to the automatic supply of optimal parameter values by the PSO algorithm to the ROHT algorithm.

f) Run the Main PSO: This stage involves running the main PSO loop to obtain the current position of the particles, updating the particles and moving the particles to the new position at every iteration(s).

C. Empirical Method of Analysis: We analyzed and compared our model with the non-optimized ROHT algorithm using the probability of detection, $\mathrm{P}_{\mathrm{D}}$; and the false alarm probability, $\mathrm{P}_{\mathrm{FA}}$ statistically described in (7) and (8).

$$
\begin{aligned}
& P D=\operatorname{Pr}(Y(n)>\gamma \mid H 1), \quad n=1,2, \ldots, N \\
& P F A=\operatorname{Pr}(Y(n)>\gamma \mid H 0), \quad n=1,2, \ldots, N
\end{aligned}
$$

Where $Y(n)$ denotes the signal spectral values for different frequency index, $n=1,2 \ldots N$, and $\gamma$ is the threshold value typically estimated by the adaptive threshold technique (ATT), $H 0$ is the null hypothesis describing the noise only spectral, and $H 1$ is the alternate hypothesis describing the signal plus noise spectral. We computed (7) and (8) following Fawcett's empirical approach [15].
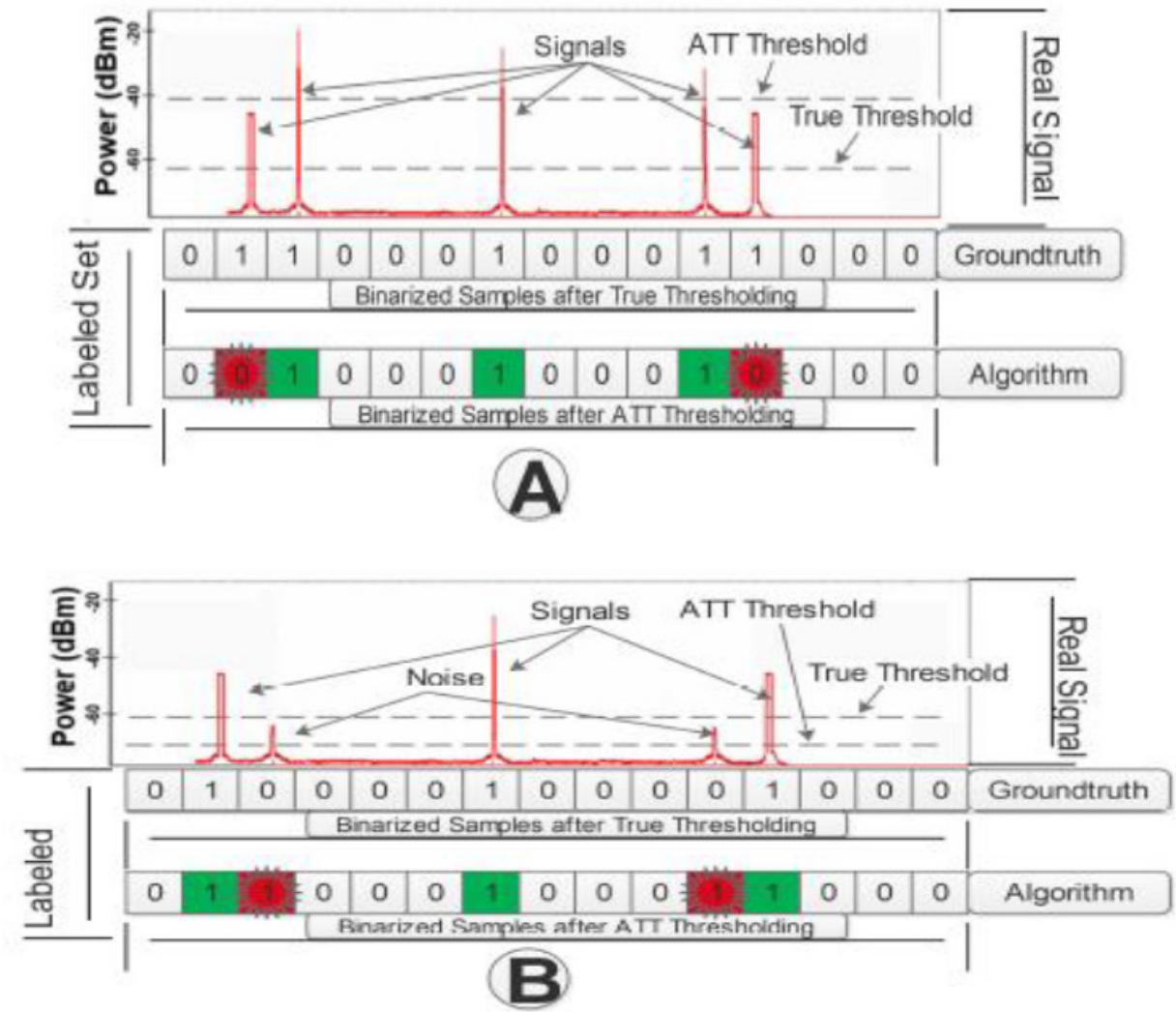

Figure 5: Method of Labeling and Analysis showing different errors: (A) Missed Detection, and (B) False alarms 
An illustration of our approach is presented in figure 5. Essentially, each dataset considered in our test cases were labeled via a binary approach as the ground truth using an a priori known true threshold value (see figure 5). In this case, samples above the true threshold are true signals and labeled as ones, while samples below the true threshold are noise samples and thus labeled as zeros. This labeling process for the ground truth was carried out for all dataset used in this work. Thereafter, each dataset was relabeled in a binary fashion based on the threshold values computed by the respective ATTs. Essentially, a missed detection error is declared if the labeled ground truth is 1 and the ATT labeled dataset is 0 , while a false alarm is declared if the labeled ground truth is 0 and the ATT labeled dataset is 1 (false positive). Furthermore, a true detection is declared when values in the both the ground truth and the ATT labeled dataset are 1 (true positive). Consequently, by using the knowledge of the confusion matrix and Fawcett's algorithm in[15], the probability of detection per dataset was computed using;

$$
P D=\frac{\emptyset}{\rho}
$$

Where $\varnothing$ denotes the total number of true positives (truly detected signal samples) if $Y(n)>\gamma \mid H_{1}$, and $\rho$ is the total number of actual true signal samples (obtained as the total number of ones in the ground truth). The false alarm probability per dataset was computed using,

$$
P F A=\frac{\varphi}{\eta}
$$

Where $\varphi$ denotes the total number of false positives (falsely detected signal samples) if $Y$ $(n)>\gamma \mid H_{0}$, and $\eta$ is the total number of noise samples (obtained as the total number of zeroes in the ground truth). In addition, rather than employing the well-known Receiver Operating Characteristic (ROC) curve (that is, $P_{D} v S P_{F A}$ ) as in [15], we generated the curves of the probability of false alarm and detection against respective thresholds, that is $P_{D}, P_{F A} v s \gamma$ as a means to easily extract the corresponding point performance of each algorithm (as tabulated in the results section).

\section{ViI. RESUlTS AND DisCUSSIONS}

In this section, we present the evaluation results for the PSO-ROHT algorithm alongside with the non-optimized ROHT algorithm for comparison. The two algorithms were trained extensively over several simulated noises only signals with varying noise uncertainty conditions, to ascertain the parameter values that would give the optimal performance for each algorithm. In this case the false alarm rate is utmost concern to the CR engineer. After the training, the two algorithms were then tested with noise plus signal datasets with varying SNR level and compared.

From the training, the standard deviation coefficient and the stopping criteria for the nonoptimized ROHT were conditioned for $z$-value $=2.5$ and $\beta=0.5$ respectively, while the PSOROHT parameters were conditioned for $P=50$, iter_num $=50$, dim $=2, C=0.6, W=0.3$, runs $=2, z_{-} u p=3.0, z_{-}$down $=2.3$, stop_up $=1$, stop_down $=0.01$. Where $\mathrm{P}=$ number of particles, iter_num $=$ number of iterations, $\operatorname{dim}=$ dimensions, that is number of parameters to optimize, $\mathrm{C}=$ weight of local and global information, $\mathrm{W}=$ inertia, $z_{-} u p=$ upper bound of $\mathrm{z}-$ value, $z_{-}$down $=$lower bound of $z-$ value, stop_up $=$ upper bound of stop criteria, stop_down $=$ lower bound of stop criteria. These values above were achieved based on false alarm probability of less than $5 \%$, under the various noise uncertainty levels considered for the ROHT and PSO-ROHT algorithm. These values were noted to provide the best performance for the algorithms and were not changed during the testing phase, which models a real life deployment scenario. 
A. Performance of the algorithms in the noise only condition, $\mathbf{H}_{0}$ :To determine the false alarm rate and show how the algorithms perform in a noise only condition, we constructed a spectrum containing only noise by simulating an Additive White Gaussian Noise(AWGN) sample $\operatorname{set}(\mathrm{N}=250)$. A sample image of the spectral (a single sweep) is shown in figure 6 . The results show that false alarm rate $P_{F A}$ of less than $5 \%$ was maintained by the two algorithms, but better estimated threshold was produced by the PSO-ROHT at each varied condition. This can be clearly seen in table 3 . This shows that our new PSO-ROHT model, continued changing its parameter values with regards to the range of the limits that it was given to get the optimal threshold value as against the non-optimized ROHT algorithm that has its parameter values fixed at $(\mathrm{z}$-value $=2.5$ and $\beta=0.5)$. Also, the PSO-ROHT achieved this low false alarm rate $\left(P_{F A}=5 \%\right)$ in an automatic manner without the need for manual pre-configuration of its parameter values. Going by the IEEE 802.22 standard (where $P_{F A}<10 \%$ ), it is clear that the PSO-ROHT having $P_{F A}$ $=5 \%$ met the standard.
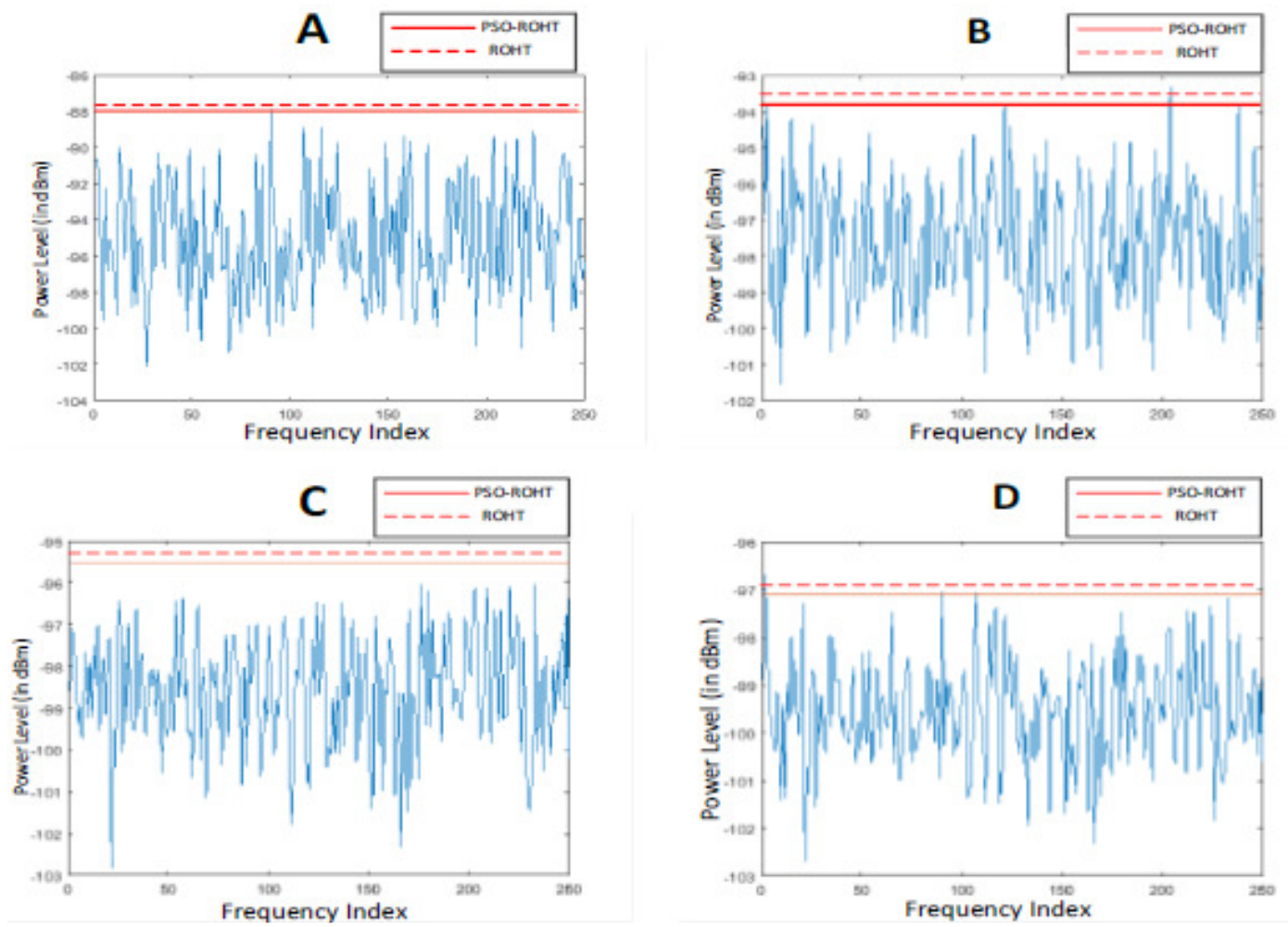

Figure 6: Threshold Computation for PSO-ROHT and non-optimized ROHT in AWGN noise only condition (A) $10 \mathrm{~dB}(\mathrm{~B}) 5 \mathrm{~dB}(\mathrm{C}) 3 \mathrm{~dB}(\mathrm{D}) 1 \mathrm{~dB}$ 
Table 3: Threshold computation for PSO-ROHT and non-optimized ROHT in noise only condition

\begin{tabular}{|c|c|c|c|c|c|c|}
\hline $\begin{array}{l}\text { Noise } \\
\text { power }\end{array}$ & $\begin{array}{l}\text { ROHT threshold } \\
\text { (in } \mathrm{dBm} \text { ) }\end{array}$ & $\begin{array}{l}\text { PSO-ROHT } \\
\text { threshold (in } \\
\mathrm{dBm} \text { ) }\end{array}$ & $\begin{array}{l}\text { PSO-ROHT } \\
P_{F A}(\%)\end{array}$ & $\begin{array}{l}\text { ROHT } \\
P_{F A}(\%)\end{array}$ & $\begin{array}{l}\text { PSO-ROHT } \\
\text { final } \\
\text { parameter } \\
\text { values }\end{array}$ & $\begin{array}{l}\text { ROHT final } \\
\text { parameter } \\
\text { values }\end{array}$ \\
\hline $10 d B$ & -87.4461 & -88.0045 & 3.1 & 2.8 & $\begin{array}{l}Z=2.3047 \\
\beta=0.4233\end{array}$ & $\begin{array}{l}Z=2.5 \\
\beta=0.5\end{array}$ \\
\hline $5 d B$ & -93.4831 & -93.8001 & 4.1 & 4.0 & $\begin{array}{l}Z=2.3030 \\
\beta=0.0509\end{array}$ & $\begin{array}{l}Z=2.5 \\
\beta=0.5\end{array}$ \\
\hline $3 d B$ & -95.2903 & -95.5311 & 2.8 & 2.8 & $\begin{array}{l}Z=2.6307 \\
\beta=0.1468\end{array}$ & $\begin{array}{l}Z=2.5 \\
\beta=0.5\end{array}$ \\
\hline $1 d B$ & -96.8832 & -97.0894 & 3.7 & 2.9 & $\begin{array}{l}Z=2.3248 \\
\beta=0.7845\end{array}$ & $\begin{array}{l}Z=2.5 \\
\beta=0.5\end{array}$ \\
\hline
\end{tabular}

B. Performance of the algorithms in the signal plus noise condition, $\mathrm{H}_{1}$ : To demonstrate the performance of ROHT and PSO-ROHT algorithms under different SNR conditions, we simulated an FM and an Orthogonal Frequency Division Multiplexing (OFDM) signal, and we varied the signal strength relative to a fixed noise level. The SNR was reduced from a high SNR level(SNR $=10 \mathrm{~dB}$ ) to a low SNR level of $1 \mathrm{~dB}$. In this work, SNR of OdB was not considered, because it means that the signal is totally buried in the noise. It is noted in [5], that sensing below SNR = OdB is a difficult task for an ED in CR especially when the ED has no knowledge of the noise floor nor the PU's frequency. The performances of the two algorithms for each SNR condition on different datasets are presented in Figure $7-8$ for FM and OFDM signals respectively. Also, for clarity, the results of the estimated threshold, $P_{D}$ and $P_{F A}$ are presented in Table $4-5$. From the table it can be seen that the PSO-ROHT algorithm continues to adapt the parameter values, so as to compute the optimal threshold.

\section{FM signals detection}

The performance of the two algorithms for each SNR condition on FM signal datasets is presented in Figure 7; 

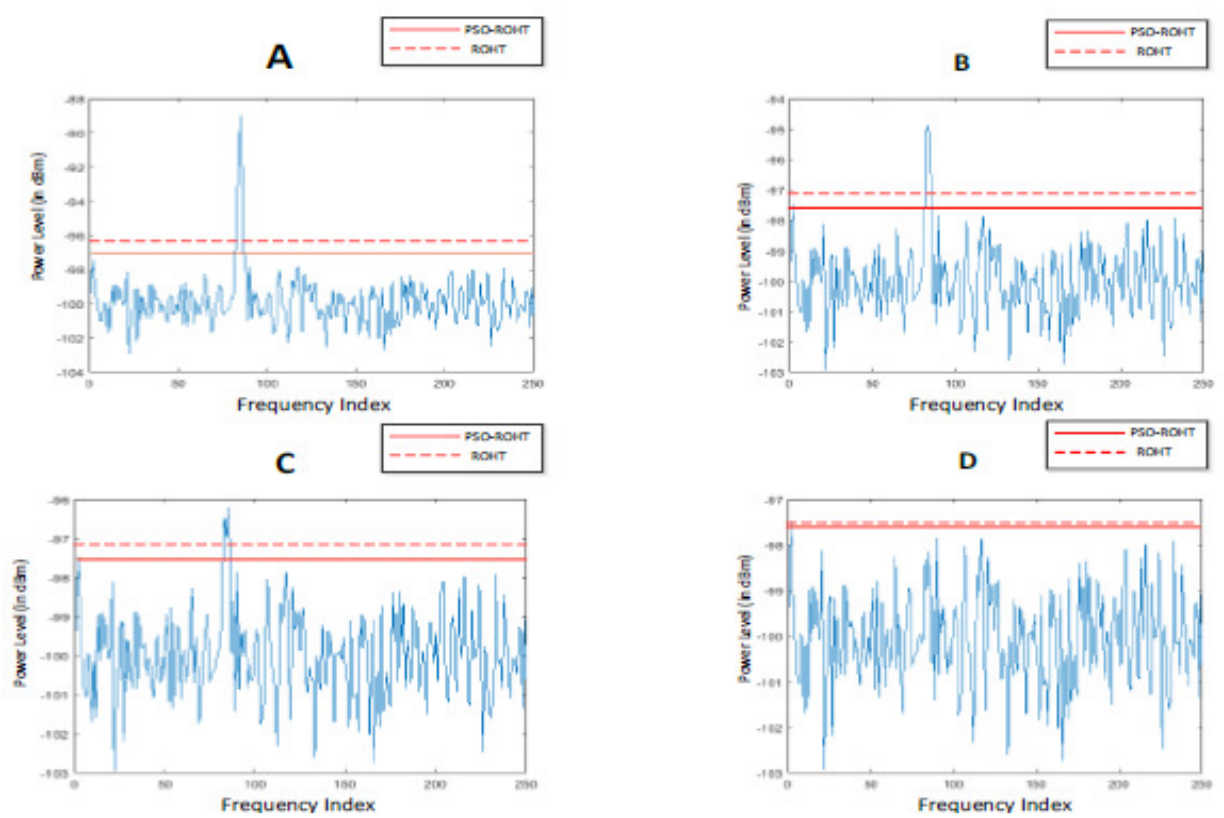

Figure7: Threshold computation for PSO-ROHT and non-optimized ROHT on signal plus noise condition for FM signal (A) $\mathrm{SNR}=10 \mathrm{~dB}$ (B) $\mathrm{SNR}=5 \mathrm{~dB}$ (C) $\mathrm{SNR}=3 \mathrm{~dB}$ (D) $\mathrm{SNR}=1 \mathrm{~dB}$.

\begin{tabular}{|c|c|c|c|c|c|c|c|c|}
\hline SNR & $\begin{array}{c}\text { ROHT } \\
\text { threshold } \\
\text { (in dBm) }\end{array}$ & $\begin{array}{c}\text { PSO-ROHT } \\
\text { Threshold } \\
\text { (in } \mathrm{dBm} \text { ) }\end{array}$ & $\begin{array}{l}\text { ROHT } \\
P_{D}(\%)\end{array}$ & $\begin{array}{l}\text { PSO- } \\
\text { ROHT } \\
P_{D}(\%)\end{array}$ & $\begin{array}{l}\text { ROHT } \\
P_{F A}(\%)\end{array}$ & $\begin{array}{l}\text { PSO- } \\
\text { ROHT } \\
P_{F A}(\%)\end{array}$ & $\begin{array}{c}\text { Final } \\
\text { parameter } \\
\text { values for } \\
\text { PSO-ROHT }\end{array}$ & $\begin{array}{c}\text { Final } \\
\text { parameter } \\
\text { values for } \\
\text { ROHT }\end{array}$ \\
\hline $10 d B$ & -96.2400 & -97.5000 & 90.53 & 96.23 & 0 & 0 & $\begin{array}{l}Z=2.3919 \\
\beta=0.9236\end{array}$ & $\begin{array}{l}Z=2.5 \\
\beta=0.5\end{array}$ \\
\hline $5 d B$ & -97.1054 & -97.6129 & 80.33 & 93.56 & 0 & 0 & $\begin{array}{l}Z=2.3600 \\
\beta=0.0235\end{array}$ & $\begin{array}{l}Z=2.5 \\
\beta=0.5\end{array}$ \\
\hline $3 d B$ & -97.2635 & -97.6573 & 70.67 & 90.81 & 0 & 0 & $\begin{array}{l}Z=2.4681 \\
\beta=0.0351\end{array}$ & $\begin{array}{l}Z=2.5 \\
\beta=0.5\end{array}$ \\
\hline $1 d B$ & -97.4994 & -97.6270 & 0 & 0 & 0 & 0 & $\begin{array}{l}Z=2.4018 \\
\beta=0.5429\end{array}$ & $\begin{array}{l}Z=2.5 \\
\beta=0.5\end{array}$ \\
\hline
\end{tabular}

Table 4: Threshold computation for PSO-ROHT and non-optimized ROHT on signal plus noise condition for FM signals showing PD, PFA and parameter values.

\section{OFDM signals detection}

The performance of the two algorithms, for each SNR condition on OFDM signal dataset is presented in Figure 8 below; 
International Journal of Wireless \& Mobile Networks (IJWMN) Vol. 10, No. 6, December 2018
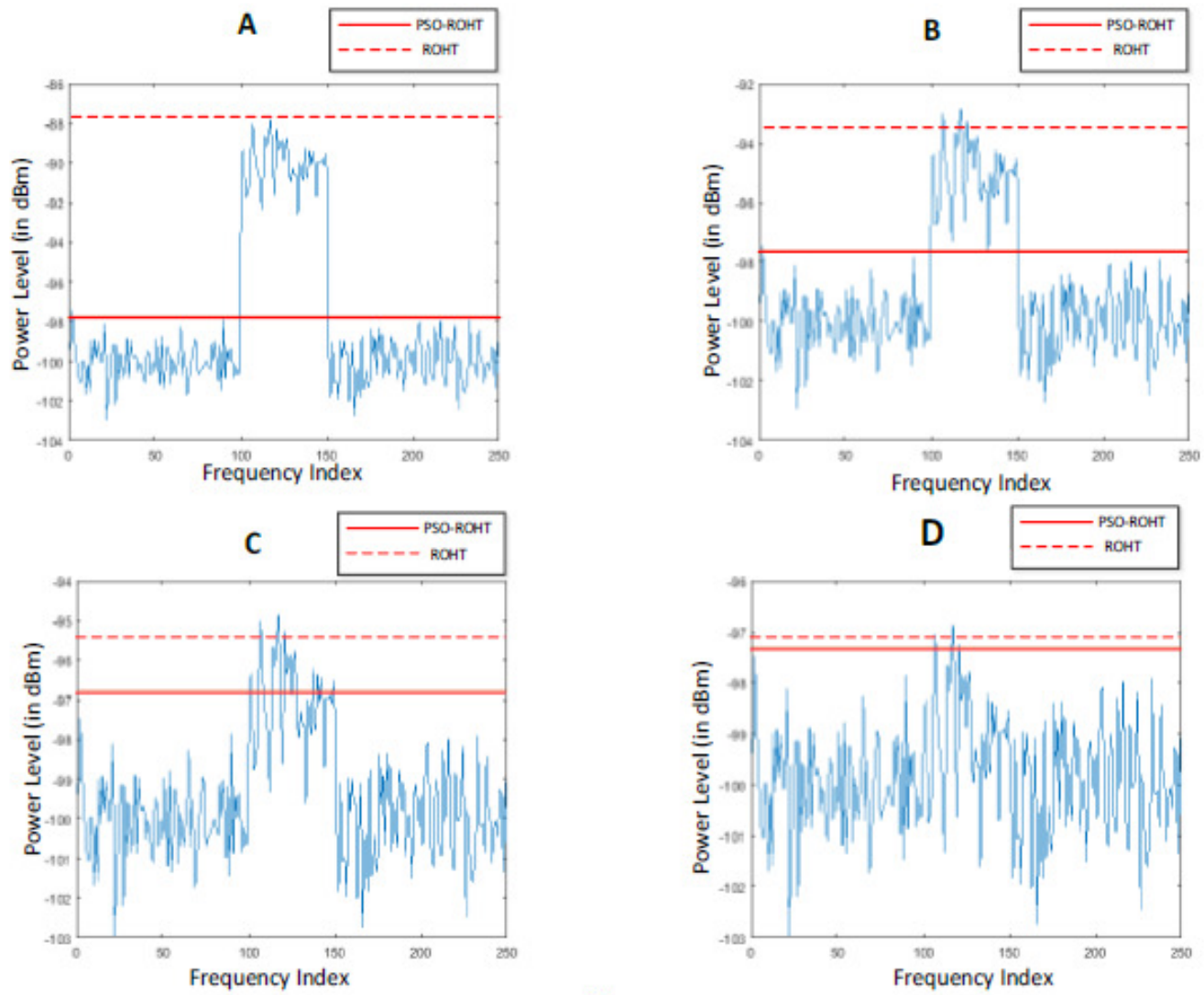

Figure 8: Threshold computation of PSO-ROHT on signal plus noise condition for OFDM signal for (A) $\mathrm{SNR}=10 \mathrm{~dB}(\mathrm{~B}) \mathrm{SNR}=5 \mathrm{~dB}(\mathrm{C}) \mathrm{SNR}=3 \mathrm{~dB}(\mathrm{D}) \mathrm{SNR}=1 \mathrm{~dB}$

\begin{tabular}{|l|l|l|l|l|l|l|l|l|}
\hline SNR & $\begin{array}{l}\text { ROHT } \\
\text { threshold } \\
\text { (in dBm) }\end{array}$ & $\begin{array}{l}\text { PSO-ROHT } \\
\text { Threshold } \\
\text { (in dBm) }\end{array}$ & $\begin{array}{l}\text { ROHT } \\
P_{D}(\%)\end{array}$ & $\begin{array}{l}\text { PSO- } \\
\text { ROHT } \\
P_{D}(\%)\end{array}$ & $\begin{array}{l}\text { ROHT } \\
P_{F A}(\%)\end{array}$ & $\begin{array}{l}\text { PSO- } \\
\text { ROHT } \\
P_{F A}(\%)\end{array}$ & $\begin{array}{l}\text { Final } \\
\text { parameter } \\
\text { values for } \\
\text { PSO-ROHT }\end{array}$ & $\begin{array}{l}\text { Final } \\
\text { parameter } \\
\text { values for } \\
\text { ROHT }\end{array}$ \\
\hline $10 d B$ & -87.6029 & -97.2239 & 1.89 & 96.23 & 0 & 0 & $\begin{array}{l}Z=1.5453 \\
\beta=0.8641\end{array}$ & $\begin{array}{l}\mathrm{Z}=2.5 \\
\beta=0.5\end{array}$ \\
\hline $5 d B$ & -93.8461 & -97.6021 & 11.32 & 96.23 & 0 & 0.8 & $\begin{array}{l}Z \\
\beta=1.5286 \\
\beta=0.8339\end{array}$ & $\begin{array}{l}\mathrm{Z}=2.5 \\
\beta=0.5\end{array}$ \\
\hline $3 d B$ & -95.7857 & -98.6631 & 11.32 & 96.23 & 0 & 8.10 & $\begin{array}{l}Z=1.4630 \\
\beta=0.0996\end{array}$ & $\begin{array}{l}\mathrm{Z}=2.5 \\
\beta=0.5\end{array}$ \\
\hline $1 d B$ & -97.1391 & -99.8125 & 3.77 & 75.47 & 0 & 42.13 & $\begin{array}{l}Z=0.0432 \\
\beta=0.5907\end{array}$ & $\begin{array}{l}\mathrm{Z}=2.5 \\
\beta=0.5\end{array}$ \\
\hline
\end{tabular}

Table 5: Threshold computation for PSO-ROHT and non-optimized ROHT on signal plus noise condition for OFDM showing PD, PFA and parameter values 


\section{ViII. CONCLUSION}

An optimized ROHT algorithm has been proposed by introducing automation in the process of parameter value estimation. This was achieved by creatively integrating the ROHT algorithm with the Particle Swarm Optimization (PSO) algorithm. The PSO algorithm in this case helps to estimate the ROHT's parameter values in an automatic manner for every singular input data set under consideration by the ED. This on-the-fly capacity of the PSO-ROHT model is new and provides efficient improvement to the common known ROHT algorithm. Several experimental use-cases were used to show that the PSO-ROHT model works better than its counterpart (the ROHT algorithm), especially in terms of detection, more especially in detecting OFDM signals. Thus, from the results obtained, it is simply concluded that the fixed and manual parameter evaluation process of the conventional ROHT algorithm can be greatly improved using the proposed model. This has extended the ROHT algorithm into a completely self-determining and blind threshold evaluation technique. The PSO-ROHT model will be of great importance to improve energy detection in CR and other likely areas of application that may need automated decision making. The PSO-ROHT model has been shown to be pertinent in quick and equally slow sensing situations, below SNR as low as $3 \mathrm{~dB}$.

Future works will consider the effect of various signal occupancy rates on the operation of both algorithms, the time involvement of the PSO-ROHT model and investigating other potential optimization methods to improve the ROHT algorithm.

\section{REFERENCES}

[1] M. Marcus, J. BUrtle, B. Franca, A. Lahjiouji and N. McNeil, "Report of the Unlicensed Devices and Experimental Licenses Working Group," 2002.

[2] M. Rouse, "Techtarget.com," November 2008. [Online]. Available: http://searchnetworking.techtarget.com/definition/cognitive-radio. [Accessed 19 June 2017].

[3] K. S. C. D. B. S. S. N. Carlos de M. Cordeiro, "IEEE 802.22: An Introduction to the First Wireless Standard based on Cognitive Radios," JCM, vol. 1, no. 1, pp. 38-47, April 2006.

[4] N. T. Ng'ethe, "An Adaptive Threshold Energy Detection Technique with Noise Variance Estimation for Cognitive Radio Sensor Networks (Doctoral dissertation, University of Cape Town)," 2015.

[5] E. N. O. A. M. A. O. U. a. M. S. A.J. Onumanyi, "A modified Otsu's algorithm for improving the performance of the energy detector in cognitive radio," AEU- International Journal of Electronics and Communication, vol. 79, pp. 53-63, 2017.

[6] S. Bames, P. v. Vuuren and B.T.Maharaj., "Spectrum occupancy investigation: Measurements in South Africa," ELSEIVIER, vol. 46, no. 9, pp. 3098-3112, 2013.

[7] S. V. Vaseghi, Advanced Digital Signal Processing and Noise Reduction, WILEY, 2008.

[8] A. M. W. a. G. J. M. D. Datla, " A Spectrum Surveying Framework for Dynamic Spectrum Access Networks," IEEE, vol. 58, no. 8, pp. 4158 - 4168, 2009.

[9] D. D. V. P. P. K. a. G. J. M. F. Weidling, "A framework for R.F. spectrum measurements and analysis," in New Frontiers in Dynamic Spectrum Access Networks, 2005. DySPAN 2005. 2005 First IEEE International Symposium on, Baltimore, MD, USA, USA, 2005.

[10] A. M. W. J. M. Dinesh Datla, "A Spectrum Surveying Framework for Dynamic Spectrum Access Networks," IEEE Transactions on Vehicular Technology, vol. 58, no. 8, pp. 4158 - 4168, 2009. 
International Journal of Wireless \& Mobile Networks (IJWMN) Vol. 10, No. 6, December 2018

[11] J. Mitola and G. Q. Maguire, "Making Software Radios More," IEEE Personal Communications , vol. 6, no. 4, pp. 13 - 18, 1999.

[12] W.-Y. L. K. R. C. Ian F. Akyildiz, "www.soebooks.com," 14 January 2009. [Online]. Available: http://www.soebooks.com/06/CRAHNs-Cognitive-radio-ad-hoc-networks.html. [Accessed 21 February 2018].

[13] A. Gorcin, K. A. Qaraqe, H. Celebi and H. Arslan, "An Adaptive Threshold Method for Spectrum Sensing in Multi - Channel Cognitive Radio Networks," in IEEE 17th International Conference on Telecommunications, Doha, Qatar, 2010.

[14] N. Otsu, "A Threshold Selection Method from Gray-Level Histograms," IEEE, vol. 9, no. 1, pp. 6266, 1979.

[15] T. Fawcett, "An introduction to ROC analysis," ELSEVIER, vol. 27, no. 8, pp. 861-874, 2006. 